\title{
RF Negative Ion Source Development at IPP Garching
}

\author{
W. Kraus, M. Berger, S. Christ- Koch, H.D. Falter, U. Fantz, P. Franzen, \\ M. Fröschle, B. Heinemann, S. Leyer, P. McNeely, R. Riedl, \\ E. Speth, D. Wünderlich
}

Max-Planck- Institut für Plasmaphysik, Boltzmannstr. 2, D-85748, Garching, Germany

\begin{abstract}
IPP Garching is heavily involved in the development of an ion source for Neutral Beam Heating of the ITER Tokamak. RF driven ion sources have been successfully developed and are in operation on the ASDEX- Upgrade Tokamak for positive ion based NBH by the NB Heating group at IPP Garching. Building on this experience a RF driven $\mathrm{H}^{-}$ion source has been under development at IPP Garching as an alternative to the ITER reference design ion source. The number of test beds devoted to source development for ITER has increased from one (BATMAN) by the addition of two test beds (MANITU, RADI). This paper contains descriptions of the three test beds. Results on diagnostic development using laser photodetachm ent and cavity ringdown spectroscopy are given for BATMAN. The latest results for long pulse development on MANITU are presented including the to date longest pulse (600 s). As well, details of source modifications necessitated for pulses in excess of $100 \mathrm{~s}$ are given. The newest test bed RADI is still being commissioned and only technical details of the test bed are included in this paper. The final topic of the paper is an investigation into the effects of biasing the plasma grid.
\end{abstract}

Keywords: $\mathrm{H}^{-}$, Ion Source Development, Neutral Beam Heating, ITER, Radio Frequency Ion Source

PACS: $52.50 . \mathrm{Dg}, \quad 52.50 .-\mathrm{b}, 52.59 .-\mathrm{f}, 52.70 .-\mathrm{m}$, 52.80.Pi

\section{INTRODUCTION}

There has been a significant step forward in realizing the dream of fusion power, ITER has been approved and it is hoped that construction of the machine will begin in the near future. Neutral beam heating has been a key component of all successful fusion machines to date and there is no reason to believe this will not be true of ITER itself, but the ITER plasma density makes it impossible to use the reliable and mature technology of positive ion based neutral beams. Negative Ion based neutral beam heating has been used on only two machines in the world [1,2] and despite more than two decades of effort remains a technical challenge. As the neutral beam power required for ITER (33 MW) is a significant portion of the total heating power available for ITER ensuring that the beams are in reliable operation from the start of plasma operation is a high priority for both ITER and the laboratories involved in the neutral beam heating ITER task. 
For the past several years work under an EFDA contract has been ongoing at IPP Garching to develop an RF driven negative hydrogen ion source. The goal of this work was to demonstrate the suitability of an RF driven source to meet the ITER design requirements[3]. Significant progress towards that goal has been made since the last PNNIB meeting in Paris and table 1 summarizes the current situation.

This paper will provide an overview of the current status of research at IPP Garching focusing on the current tasks of the three test beds dedicated to negative ion source development: BATMAN (Bavarian Test Machine for Negative Ions), MANITU (Multi-Ampere Negative Ion Test Unit) and the newest test bed RADI that is named after the vacuum box for the Radi al Injector for the W7AS Stellerator it is mounted on. The test beds themselves will be described, the goals and current status of the experimental campaigns being carried out detailed, and lastly a few of the significant and interesting new experimental results given.

Lastly, the question of plasma grid biasing, a topic that should be of general interest to the community, will be discussed in greater detail. The development of biasing at IPP will be given, along with the results of the latest investigations. This paper will concern itself mainly with giving the experimental background to this question compared to the companion paper that presents modelling results [4]. The question of what form the plasma takes in front of the grid will be discussed as it pertains to the situation with biasing.

\section{IPP GARCHING TEST BEDS}

\section{Test Bed BATMAN}

BATMAN (Bavarian Test Machine for Negative Ions) has been in use for negative ion development the longest and has been described previously[5]. This test stand only allows testing of sources with reduced extraction area $\left(\approx 70 \mathrm{~cm}^{2}\right)$ due to the saturation of the titanium pumps, and for limited duration shots $(<4 \mathrm{~s}$ with extraction due to the voltage divider employed or $<10 \mathrm{~s}$ plasma only due to the limitation of the $\mathrm{RF}$ generator). Shot to shot repletion rate is relatively flexible but for a number of technical reasons is limited to a minimum time interval of $90 \mathrm{~s}$. The $1 \mathrm{MHz} R F$ generator can provide up to $120 \mathrm{~kW}$ of power (as measured at the generator). Using the main high voltage supplies of IPP up to $22 \mathrm{kV}$ can be applied to the source, split between extraction and acceleration by the voltage divider. A gas handling system provides one or two different gases to the source at variable flow rates allowing for simple and reproducible pressure scans. The beam current and its profile are measured on a water- cooled calorimeter. The ion current on the calorimeter can be determined either by fitting the signals from the thermocouples or else by using the increase in water temperature and flow rate. Both methods are in agreement with each other and in general $>75 \%$ of the current density determined from the currents flowing from the power supply is found on the calorimeter. The overall current accountability is better than 90\%. The whole test bed is computer controlled via a Siemens S7 control system, and all relevant data signals are automatically logged via a combined CAMAC and $\mu$ MUSIC DAQ.

As all ITER relevant parameters that could be confirmed on BATMAN have been [3] the test stand has turned to other matters in the past year. Currently BATMAN uses the type VI-1 ion source [6] and experiments with it are performed: to further optimize specific aspects of $\mathrm{H}^{-}$production and to develop diagnostic techniques for use on the other test stands. Optimization studies at BATMAN are critical to the success of MANITU as changes to the source configuration can be done in a much 
shorter time frame on BATMAN. This reduced time "cost" allows for freer investigations without so much concern for the consequences of a failed line of experimental inquiry.

As the RADI test bed has no extraction system it is critical to calibrate the diagnostics used on the source there so that reasonable estimates of expected source performance may be made. For the purposes of determining expected $\mathrm{H}^{-} / \mathrm{D}^{-}$yields two diagnostics in particular are important: laser photo- detachment (LPD)[7] and cavit y-ringdown- spectroscopy (CRS)[8]. The laser photo- detachment system is currently being optimized and the cavity-ringdown- spectroscopy system is ready for deployment on RADI.

The principles of operation of the CRS system and specific details relating to its implementation on BATMAN are given in [9]. The line averaged $\mathrm{H}^{-}$density is given by:

$$
\bar{n}_{H^{-}}=\frac{1}{\sigma_{H-} c}\left(\frac{1}{\tau^{\prime}}-\frac{1}{\tau_{o}}\right) \frac{d}{L}
$$

Where: $\bar{n}_{H^{-}}$is the line averaged $\mathrm{H}^{-}$density, $\sigma_{\mathrm{H}-}$ is the absorption cross- section $(3.5 \mathrm{x}$ $\left.10^{-21} \mathrm{~m}^{2}\right), \tau_{0}$ is the decay constant of an empty cavity, $\tau$ ' is the decay constant of the cavity with plasma, $d$ is the length of the plasma, and $L$ is the separation of the mirrors.

The CRS system has provided results (see fig. 1) that clearly indicate that there is a strong correlation between the measured $\mathrm{H}^{-}$density and the extracted ion current density. The measured densities are also in reasonable (better then within a factor of two) agreement with the results of a simple freefall calculation for the $\mathrm{H}^{-}$density required for a particular extracted ion current density and assumed ion temperature. The good qualitative agreement with the extracted ion current density means that the density measured by the diagnostic can be used to estimate the expected current density extractable from the source on RADI. CRS is superior to the alternative method developed at IPP that uses the ratio of the $\mathrm{H}_{\gamma}$ to $\mathrm{H}_{\beta}$ lines [10] in that it is a direct measurement whose result is dependant only on the accuracy of absorption cross-section and the plasma size and is not dependant on modelling or any assumptions on plasma properties. The primary disadvantage of the CRS system is that it is a line averaged density measurement. This system is the only one currently in operation on an ion source used for fusion development.

The LPD system complements the CRS system by allowing spatially resolved measurement of the $\mathrm{H}^{-}$density. It has proven challenging to develop the LPD system mainly due to the use of an RF driven source. The frequency of the RF signal that is present in the plasma is approximately the same as that of the photo- detachment pulse itself. This prevents the use of a simple filter to remove the RF interference. To reduce external pick up of the RF has required that particular attention has been paid to all aspects of the signals (grounding of cables, connectors, etc). To compensate for the unavoidable plasma pickup the laser pulse itself is triggered $90^{\circ}$ out of phase with the RF signal. The phase angle can be manually adjusted. The RF signal is then shifted and subtracted from the signal collected just after the laser pulse allowing for a relatively clear signal to be obtained. Shown in Fig. 2 are the results of a scan over the plasma grid giving the ratio of the $\mathrm{H}^{-}$density to the electron density.

The CRS, LPD and Langmuir probe systems used on BATMAN (or any other test bed) are designed to operate even when the source is at high potential. This allows for simultaneous measurements of plasma parameters and the extracted $\mathrm{H}^{-}$ion beam both 
simplifying and improving the ease of developing models and other theoretical analysis.

\section{Test Bed MANITU}

The MANITU test stand is dedicated to showing the two aspects of the ITER requirements that are still outstanding. The first is extraction of the ion beam over a reasonable size and the second aspect is to demonstrate long pulses with the RF source. Going from BATMAN with a $70 \mathrm{~cm}^{2}$ extraction area and a duty cycle of around $2 \%$ to $174 \mathrm{~cm}^{2}-188 \mathrm{~cm}^{2}$ extraction area and essentially $\mathrm{CW}$ operation has presented several serious technical challenges.

The test bed vacuum is supplied by a bath cryogenic pump developed in a collaboration with FZ Karlsruhe[11]. Operational experience has shown the pumping speed is sufficient to allow operation at all planned gas flows. The pump is regenerated daily to prevent build up of hydrogen. A new CW high voltage power supply was installed and commissioned in the 2005 summer maintenance period. The extraction and acceleration voltage (max. $15 \mathrm{kV}$ and $35 \mathrm{kV}$ respectively) is controlled by a unique dual tetrode system developed at IPP Garching. A long pulse capable calorimeter built from 4 ASDEX target panels (water cooled) with a calorimeter cross between them is currently being commissioned. For MANITU we quote only electrically measured ion currents. According to the results with the short pulse calorimeter the calorimetric current is expected to be lower by a factor 0.75 to 0.85 . Either water tanks or polyethylene to reduce the neutron flux expected to be present during deuterium operation provides radiation shielding of the test bed (Fig. 3). Three neutron monitors and one gamma ray detector are installed in the periphery of the test bed to monitor the radiation levels. The test bed is controlled by a Siemens S7 based control system and uses a $\mu$ Music based DAQ. The data from each shot is archived at the central data storage facility of IPP Garching.

The RF generator for MANITU is $1 \mathrm{MHz}$ and rated for $180 \mathrm{~kW}$ for $\mathrm{CW}$ operation. However, as the pulse length has been increased weaknesses in the design of the connections, transmission lines, and the insulating transformer have become apparent. A concerted effort has been made to improve all these aspects of the RF system. All connections are now solid strips of considerable width as opposed to cable connections and where possible straps are used instead of cables, cooling has been added where necessary. The transformer for isolating the RF generator from the source high voltage is the final component that is in need of work. The transformer is a ferrite transformer with a 3:1 winding ratio. The ferrites themselves are air-cooled and show only a modest temperature rise allowing the current design to be used for pulses of several hundred seconds. The more significant problem is damage to the insulated cables in the transformer that is occurring at certain areas where a high electrical stress is present even though the electrical potential present is significantly below the rated capability of the insulation used.

Additionally breakdowns can occur in the RF coil itself when the source filling pressure is reduced below $0.4 \mathrm{~Pa}$ or RF power increases above $75 \mathrm{~kW}$. Both of these problems seem to be due to formation of local glow discharges that gradually damage the insulation of either the coil or the transformer until a breakdown occurs. Several approaches to reduce the probability of a glow discharge developing are under study.

Significant increases in beam on time have been accomplished in the last year going from pulses of tens of seconds to pulses of over five hundred seconds (maximum pulse length $600 \mathrm{~s}$ ). In addition to those thermal aspects that had been anticipated, some of the increases in pulse duration would uncover unanticipated 
weaknesses in the current design that required revision. There have been three primary modifications to the source design added over the last year all prompted by a desire to control the temperature of specific parts of the source during long pulses better.

With the edge cooled Faraday shield the pulse length was limited to less than $40 \mathrm{~s}$, then the copper started glowing. The first improvement was an actively cooling system for the Faraday Shield that adds water- cooling channels to the wall of the shield itself rather than relying on thermal conduction to the edges of the shield. The pulse length then could be extended to approx. $200 \mathrm{~s}$ (Fig. 4). But the ion current falls with increasing time and the electron current starts to rise after a few seconds and increases by a factor 3 during the pulse. The cesium 852 emission increases in the same way. A rough estimation showed that this rise can be explained by the cesium coming off the heated plasma grid, therefore it was clear that a temperature control of the plasma grid is of great importance.

So the second modification was the addition of an air-cooled plasma grid with a extraction area slightly extended from 174 to $188 \mathrm{~cm}^{2}$. This grid uses forced air cooling during the pulse to keep the temperature of the grid constant and between pulses both reduces the air flow (by a control valve) and engages an electrical heater that serve to keep the grid at a user defined temperature. The plasma grid currently in use is a modified grid from the positive ion systems for the ASDEX Upgrade neutral beam heating ion sources. As the extraction area was greater than desired of an uncooled masking plate was installed just above the grid. After this change the currents remained more or less constant during the first $200 \mathrm{~s}$ (Fig. 5) with best results so far for the ion current approaching $4 \mathrm{~A}\left(210 \mathrm{~A} / \mathrm{cm}^{2}\right)$. Fig. 6 shows a $600 \mathrm{~s}$ pulse. Common to all pulses in excess of $200 \mathrm{~s}$ is a rising electron current with time, similar to the case with un-cooled plasma grid, but delayed by $100-200 \mathrm{~s}$. The heating of the un-cooled grid mask by the plasma is believed to be one of the causes of source degradation during long pulse operations. However, the mask allows easy variation of the extraction area to determine the optimal value. Once that is found a new air-cooled plasma grid will be fabricated with that extraction area, allowing, it is hoped, for increased pulse duration. The final improvement to the source was to provide a temperature control unit to the cooling water of the source body allowing the wall temperature to be controlled. This has proven effective in controlling caesium use in the source.

Initial results for deuterium operation on MANITU can be found in [12].

\section{Test Bed RADI}

The W7AS stellerator was decommissioned in 2002 and the injector box of the Radial Injector[13] was available to be used as the mounting and pumping system for the "Half- Sized ITER" source proposed by IPP Garching in 2003[3]. The test bed is shown in Fig. 7 and the source itself in Fig. 8. It has a width that is nearly identical to the ITER reference source but is only half as high (inner dimensions: $0.76 \times 0.8 \mathrm{~m}^{2}$ ). The RADI test bed is intended to demonstrate the plasma uniformity of a RF source of ITER dimensions, to demonstrate that the RF source is compatible with the ITER reference filter field generated by the superposition of fields created by permanent magnets and a current flowing through the plasma grid, to gain experience on driving more than one driver with a single generator, and to test an ITER- like RF circuit.

The vacuum chamber of the test bed is equipped with a Ti getter pump with a pumping speed of $160,000 \mathrm{l} / \mathrm{s}$ but with a limited duration (some $10 \mathrm{~s}$ ). As it was not intended to extract a beam from RADI and to reduce the cost of construction the 
plasma grid installed in the test bed differs from the plasma grid proposed for ITER. The grid is not equipped with extraction apertures instead it has slits with chevron baffles that give a gas flow through the source equal to that of the ITER source. The chevron's allow for adjustment of the rate of gas flow through the source to investigate its effect on the RF system and $\mathrm{H}^{-}$generation in the source. It is planned to use both hydrogen and deuterium gases on the test bed.

The test bed has two $180 \mathrm{~kW}, 20 \mathrm{~s}$ pulse duration $1 \mathrm{MHz}$ RF generators. Each generator is connected to two drivers (identical to the Type 6 driver) in series via a matching network and coaxial transmission line. As the test bed has no extraction (the source is at ground potential) it is possible to avoid the use of an isolation transformer as is used on the other test beds. This allows for the use of a RF circuit that is conceptually identical to that proposed for ITER. It is possible to install a dished plate over the RF drivers of the source and test vacuum operation in addition to operation in air.

The test bed is operated from a remote location by a control system and DAQ that uses the same hardware and similar software to what is used on MANITU. All data is stored in the central storage facility of IPP.

The magnetic filter field will be generated by a combination of permanent magnets and a current flowing through the plasma grid. The current is provided by a power supply rated for $12 \mathrm{~V}$, and $5 \mathrm{kA}$. The PG filter supply has been tested on a dummy load successfully. Samarium- cobalt permanent magnets will be used on the outside of the source to provide the static part of the magnetic filter field. An option is available to introduce additional permanent magnet equipped rods into the source to modify the filter field.

Considerable effort has gone into providing diagnostic access for optical emission spectroscopy, Langmuir probes, laser photo- detachment, and cavity ring down spectroscopy. The Langmuir probes will be of the type used on BATMAN but will have a longer stroke $(500 \mathrm{~mm})$ enabling measurements from one side of the source to the middle or from the driver to the grid. Two new 3-channel spectrometers have been commissioned and additionally up to 3 single channel spectrometers can be used to allow for multiple simultaneous measurements. A total of $16,40 \mathrm{~mm}$ diameter, ports are available for diagnostics.

As of September 2006, all services and the control system itself have been commissioned. The first plasma was generated in the bottom two drivers on 27/07/06, details of this plasma and further details of the test bed can be found in [15].

\section{BIASING THE PLASMA GRID}

It has become common practice to bias the plasma grid to suppress co-extracted electrons. The effect of biasing the plasma grid is one of the few aspects of $\mathrm{H}^{-}$ion source development that is universal; it is independent of how the source is operated (volume or production) or source of plasma (filaments or RF coil). In all cases the electron and ion current are not affected by the bias until a certain threshold is passed and then both the co-extracted electrons and the ion begin to drop. The co-extracted electron current decreases faster than the ion current does, enabling the user to control, to a degree, the ration of $\mathrm{H}^{-}$to electrons extracted from the source.

With the Type VI source at BATMAN biasing was used both with the older CEA grid system [6] and with the large area grid. As the large area grid required masking to reduce the extraction area an external plate was added over the grid to reduce the area of biased plasma grid that was exposed to the plasma as it was felt that the current would otherwise be too high for the power supply. Investigations of the effect 
of biasing the plasma grid have been carried out with the LAG on BATMAN and the results are shown in Fig. 9.

This data shows a number of interesting features. The first is that the reduction of current effect of biasing the plasma grid does not begin until one crosses a threshold. That threshold is proportional to the plasma potential of the ion source. The second is that the electrons are more strongly affected by biasing than the $\mathrm{H}^{-}$ions. It is possible to reduce the co-extracted electron current to nearly zero if you are willing to pay the price of a reduced ion current. The third is that the decrease in co-extracted electron current is exponential. The fourth is that the bias current drawn above the threshold increases linearly with increasing bias voltage. These two points make it unlikely that the bias current is composed solely of the electrons removed from the beam. The fifth is that in the case of using a current limited bias power supply in situations where the voltage limit is high but the current limit is low for that voltage setting (below what the linear increase of bias current would require) then the resultant suppression is much the same as for a voltage that corresponds to the current allowed to flow. Thus if operation in a current limited mode is desired sufficient current must be allowed to flow for the voltage applied to achieve the maximum potential biasing effect.

If one steps back from the experimental observations and considers what is observed and asks the questions "Does this function the way I would expect?" or "What is going on here?" it is difficult to escape the fact that the understanding of the situation is poor. If the plasma grid is considered as a Langmuir probe immersed in the plasma then the current characteristic observed is at variance with the typical Langmuir probe signal. If you would ask the question "If one wishes to prevent the passage of electrons through a perforated plate what polarity should the plate be biased at?" the standard answer would be negatively yet in the case of a biased plasma grid that is not the case and even more puzzling is the fact that when negatively biased there is no effect co-extracted electrons. If the $\mathrm{H}^{-}$are largely produced on the plasma grid itself then the question of how you remove them from a positively biased surface needs also to be considered, or what effect the bias has on their initial starting energy.

The question of how biasing the plasma grid reduces the co-extracted electron current is also unanswered. Clearly the nature of the plasma near the plasma grid must play a critical role in determining how the bias effect operates. However, the exact nature of that plasma is not well understood. Considerable evidence has accumulated that the plasma in front of the plasma grid may consist of a mix of positive ions, electrons and negative ions but where the negative ion density is not trivial. Also depending on the exact conditions of the plasma the mean free path of negative ions can be considerably larger than the 1 or $1.5 \mathrm{~cm}$ often assumed by the community, especially if the electron density is low and the electrons themselves are cold as then a much lower collision frequency has to be assumed. The relatively high perpendicular mobility of $\mathrm{H}^{-}$ions with respect to the filter field may also play a role. The nature of the sheath before the plasma grid may play as critical a role and its nature is equally poorly understood.

On one hand, the actual effect of biasing the plasma grid is one of the successes of the development of negative ion sources of neutral beam heating as: it does what the community wishes, it is trivial to perform and it works for everyone in the same fashion. On the other hand, it is a poorly understood phenomenon worthy of more attention then it has so far received.

\section{FUTURE PLANS}


For the test beds a full experimental program exists for the next year. The calibration of the RADI diagnostics will continue on BATMAN. A new oven will be tested that replaces the current caesium ampoule with a dispenser allowing for more control over the rate of introduction of caesium into the plasma.

The situation on MANITU looks promising and design and testing of an improved plasma grid without grid cover is planned. This coupled with a new design for the RF coil will allow the pulse length to be extended to one hour. Once this is achieved then operational studies in deuterium can begin in earnest.

Final commissioning of systems and initial experiments on RADI will begin shortly. Once this phase of the project is completed the bulk of the diagnostics can be installed and experiments begun on plasma uniformity, effect of the magnetic filter, etc. As well engineering work has begun to develop a design that will allow full extraction at the RADI test bed. The pulse length will be limited to $10 \mathrm{~s}$ but the extraction area should be approximately half that of the ITER source. 


\section{REFERENCES}

1. Y. Takeiri, et al., Nucl. Fusion 46 (2006) S199.

2. Y. Ikeda, et al., Nucl. Fusion 46 (2006) S211.

3. E. Speth, et al., Nucl. Fusion 46 (2006) S220.

4. D. Wünderlich, these proceedings.

5. P. McNeely, et al., Rev. Sci. Instrum. 77 (2006) 03A519.

6. W. Kraus, et al., Rev. Sci. Instrum. 75 (2004) 1832.

7. M. Bacal, Rev. Sci. Instrum. 71 (2000) 3981

8. A. O'Keefe and D.G. Deacon, Rev. Sci. Instrum. 59 (1988) 2544.

9. M. Berger, Cavity Ringdown Spektroskopie an Niederdruck Wasserstoffplasmen , Ph. D. Thesis, University of Augsburg, Germany, 2006.

10. U. Fantz and D. Wünderlich, submitted to the New Journal of Physics.

11. M. Dremel et al., Proceedings of the $23^{\text {rd }}$ Symposium on Fusion Technology , Venice, 2004 [Fusion Eng. Des. 74 (2005) 205].

12. P. Franzen, to be presented at the IAEA Conference, Cheng- Do, China (2006).

13. N. Rust, et al., Recent results from W7-AS with the new radial NBI injector, in: $29^{\text {th }}$ EPS

Conference on Plasma Physics and Controlled Fusion, ECA, Vol 26B, Montreux, June 17-21, (2002) 4045.

14. M. Förschle et al., to be presented at the $24^{\text {th }}$ Symposium on Fusion Technology, Warsaw, Poland (2006). 


\section{$\underline{\text { Tables }}$}

Table 1. Shown below is the current status of the development at IPP compared to the ITER requirements.

\begin{tabular}{|l|l|}
\hline \multicolumn{1}{|c|}{ ITER Requirement } & \multicolumn{1}{|c|}{ Status of Development } \\
\hline \hline Extracted Current Density: $20 \mathrm{mAcm}^{-2} \mathrm{D}^{-}$ & Demonstrated on BATMAN \\
\hline Ratio of electron to ion current: <1 & Demonstrated on BATMAN \\
\hline Pulse Duration: 3600 s & To Be Demonstrated on MANITU \\
\hline Uniform Plasma & To Be Demonstrated on RADI \\
\hline Capable of low pressure operation: $0.3 \mathrm{~Pa}$ & Demonstrated on BATMAN \& MANITU \\
\hline Can use ITER Magnetic Filter & To Be Demonstrated on RADI \\
\hline Suitable for large area extraction & Planned for an upgraded RADI test bed \\
\hline
\end{tabular}




\section{$\underline{\text { Figure Captions }}$}

Figure 1. Shown above is the line averaged $\mathrm{H}^{-}$density measured by Cavity Ringdown Spectroscopy with the simultaneously measured ion current density for two different source pressures at BATMAN. Also shown is the expected $\mathrm{H}^{-}$densities for two free fall calculations. The measurement is made $3 \mathrm{~cm}$ above the plasma grid. It is unlikely that the mean free path of $7.5 \mathrm{~cm}$ is realistic; this curve (free fall $2.3 \mathrm{eV}$ ) is intended to show that it is possible to achieve the measured relationship.

Figure 2. Shown above are the results for Laser Photo- detachment measurements on BATMAN giving the ratio of the $\mathrm{H}^{-}$density to the electron density for different positions $3 \mathrm{~cm}$ above the plasma grid. At $-6 \mathrm{~cm}$ the bias plate screening the plasma grid ends.

Figure 3. Shown above is a side view of the MANITU test stand with all major components indicated.

Figure 4. Time traces of a $180 \mathrm{~s}$ pulse showing ion current, electron current and intensity of the Cs852 emission. Pulse is performed with an edge cooled plasma grid.

Figure 5. $240 \mathrm{~s}$ beam pulse with an ion current of $3.8 \mathrm{~A}$, achieved using the actively cooled plasma grid.

Figure 6. Time traces of electron and ion current for a $600 \mathrm{~s}$ beam pulse

Figure 7. Shown above is a schematic of the test bed RADI with the major components indicated.

Figure 8. Shown above is a schematic of the "Half Sized ITER Source" installed on the RADI test bed.

Figure 9. Shown above are the results of a bias scan taken at BATMAN. The ion and electron currents as well as the measured bias current for different bias voltages are shown. The points above $26 \mathrm{~V}$ were taken with the bias power supply limited to the bias current observed. 


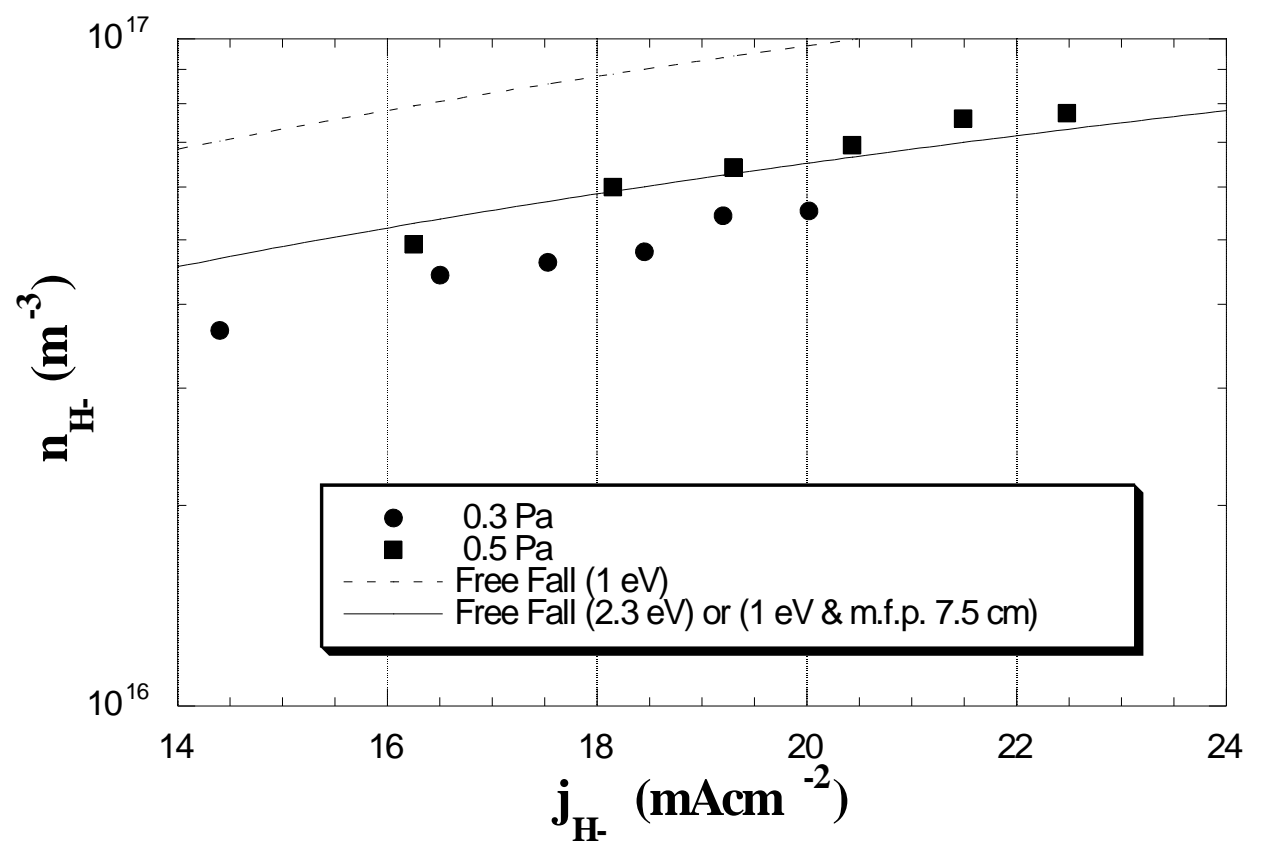

FIG. 1 


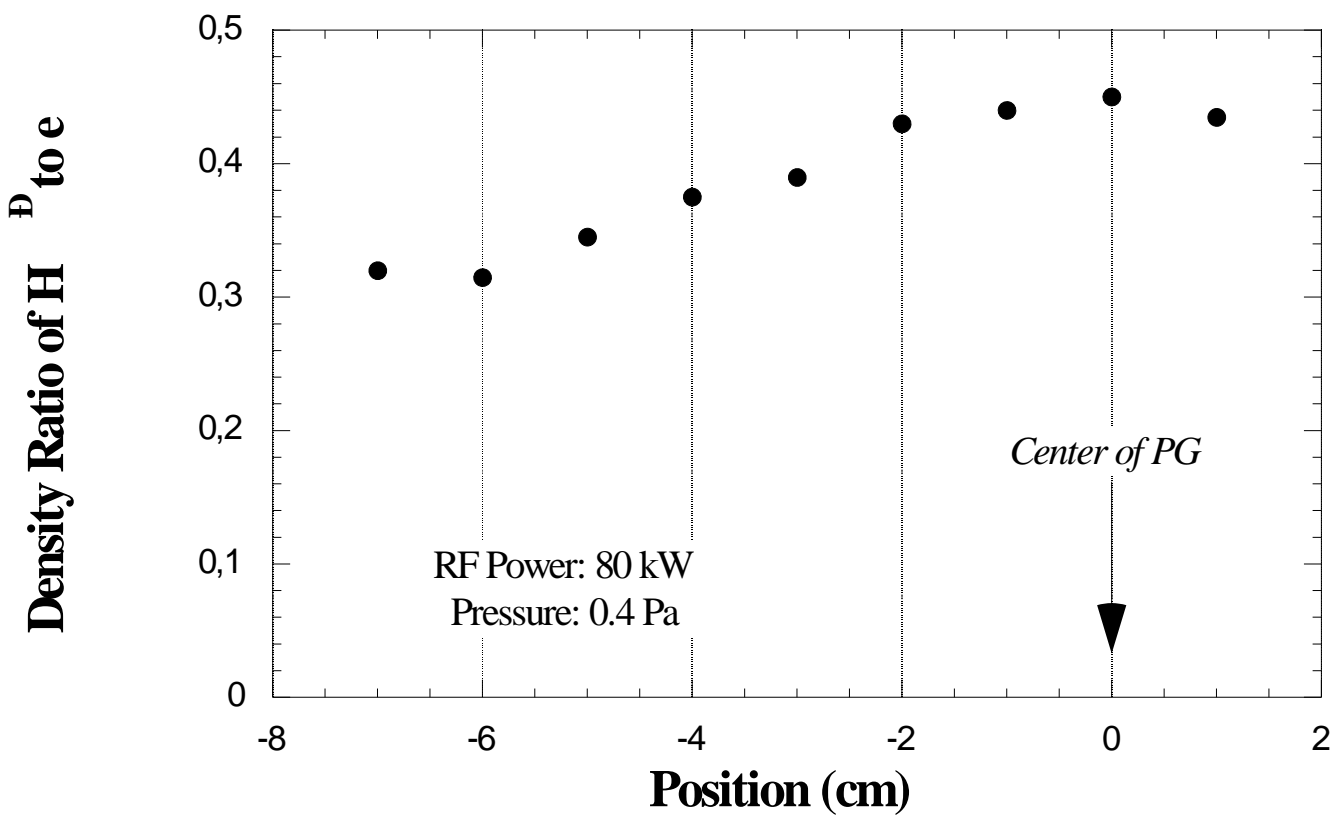

FIG. 2 


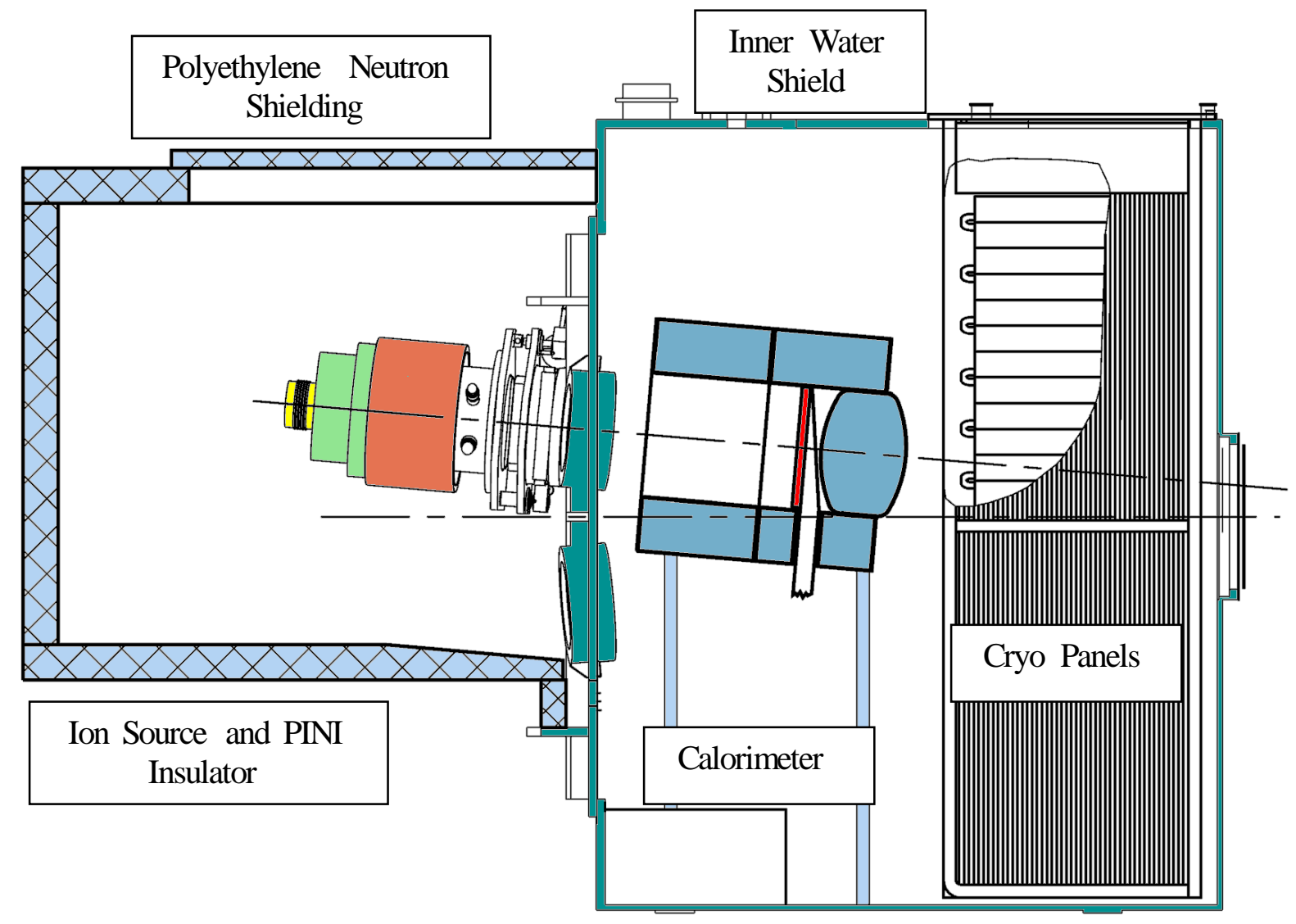

FIG. 3 


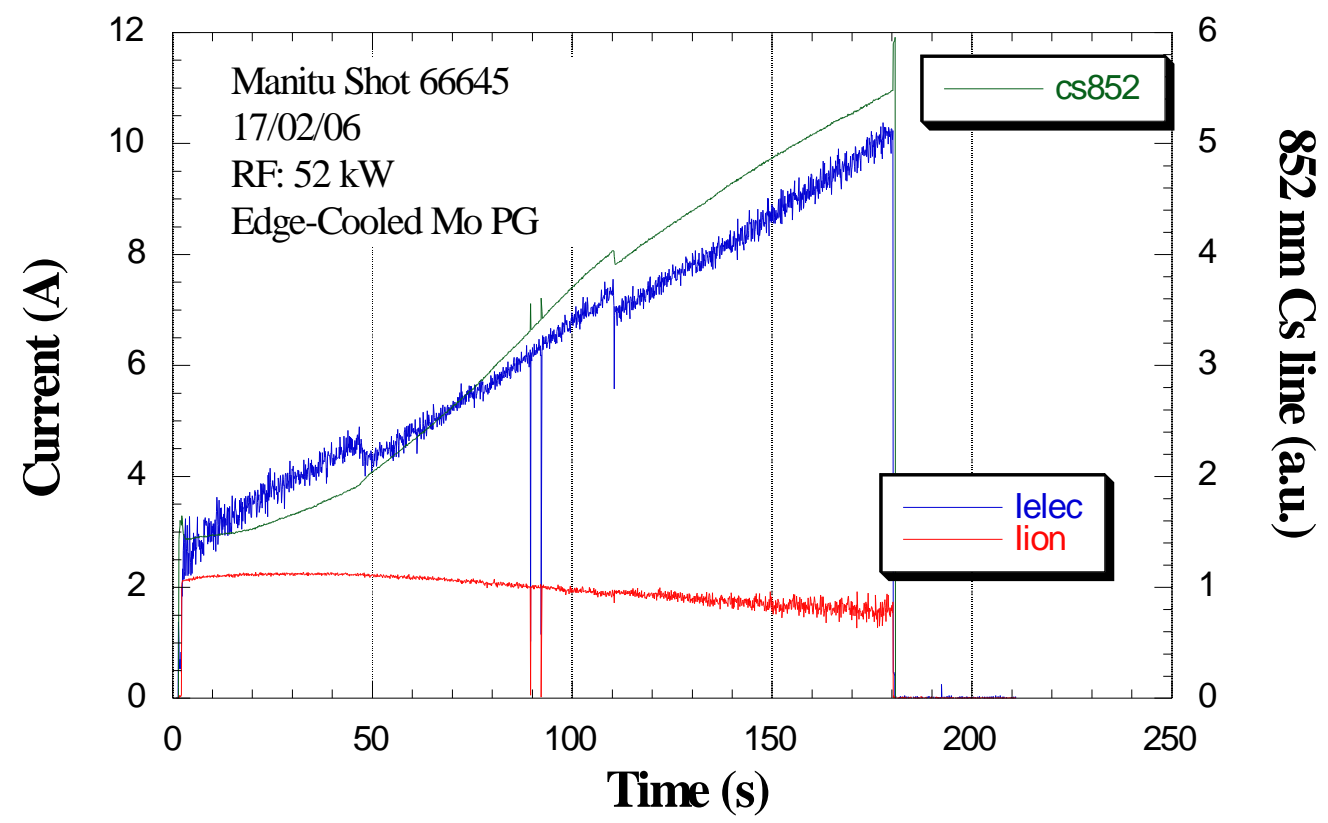

FIG. 4 


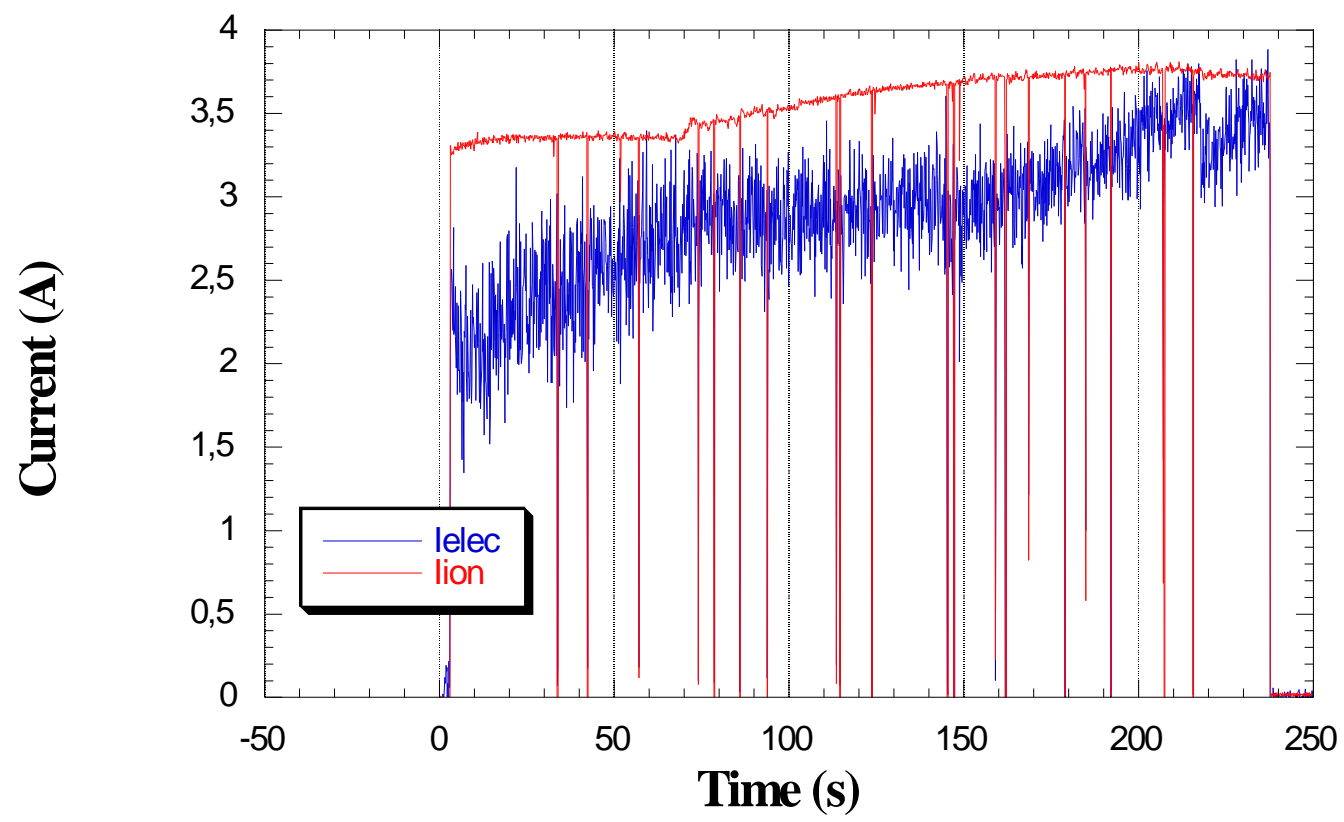

FIG. 5 


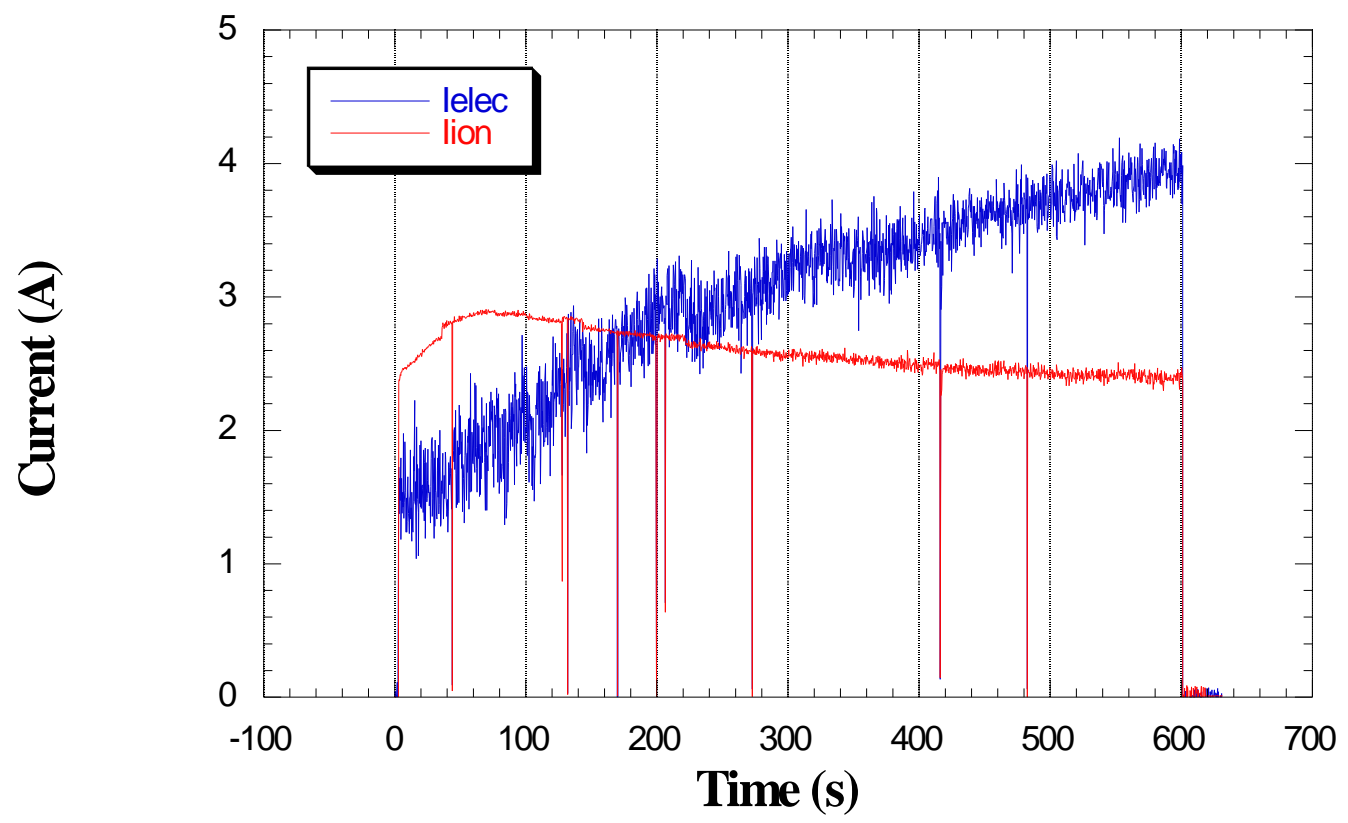

FIG. 6 


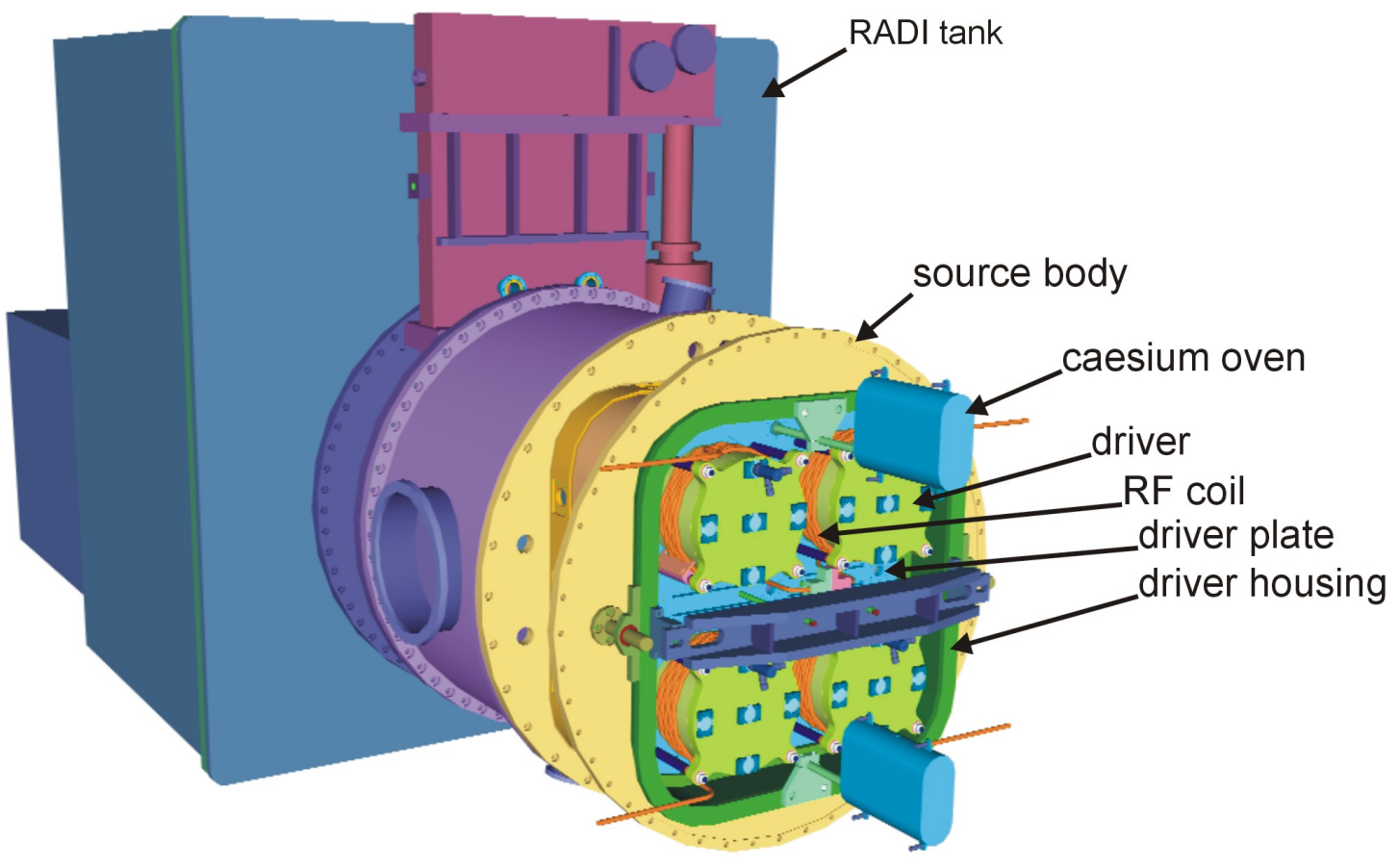

FIG. 7 


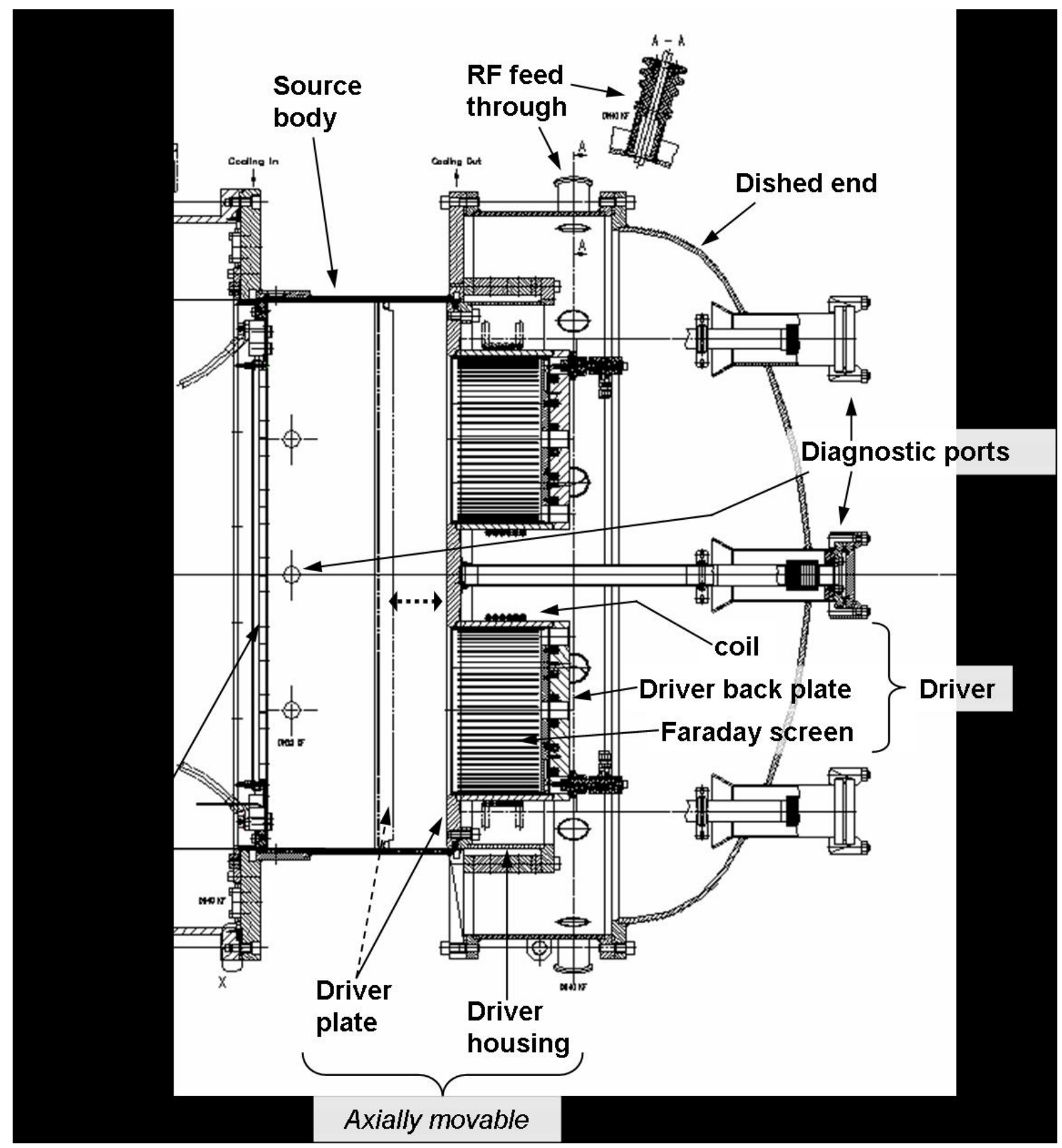

FIG. 8 


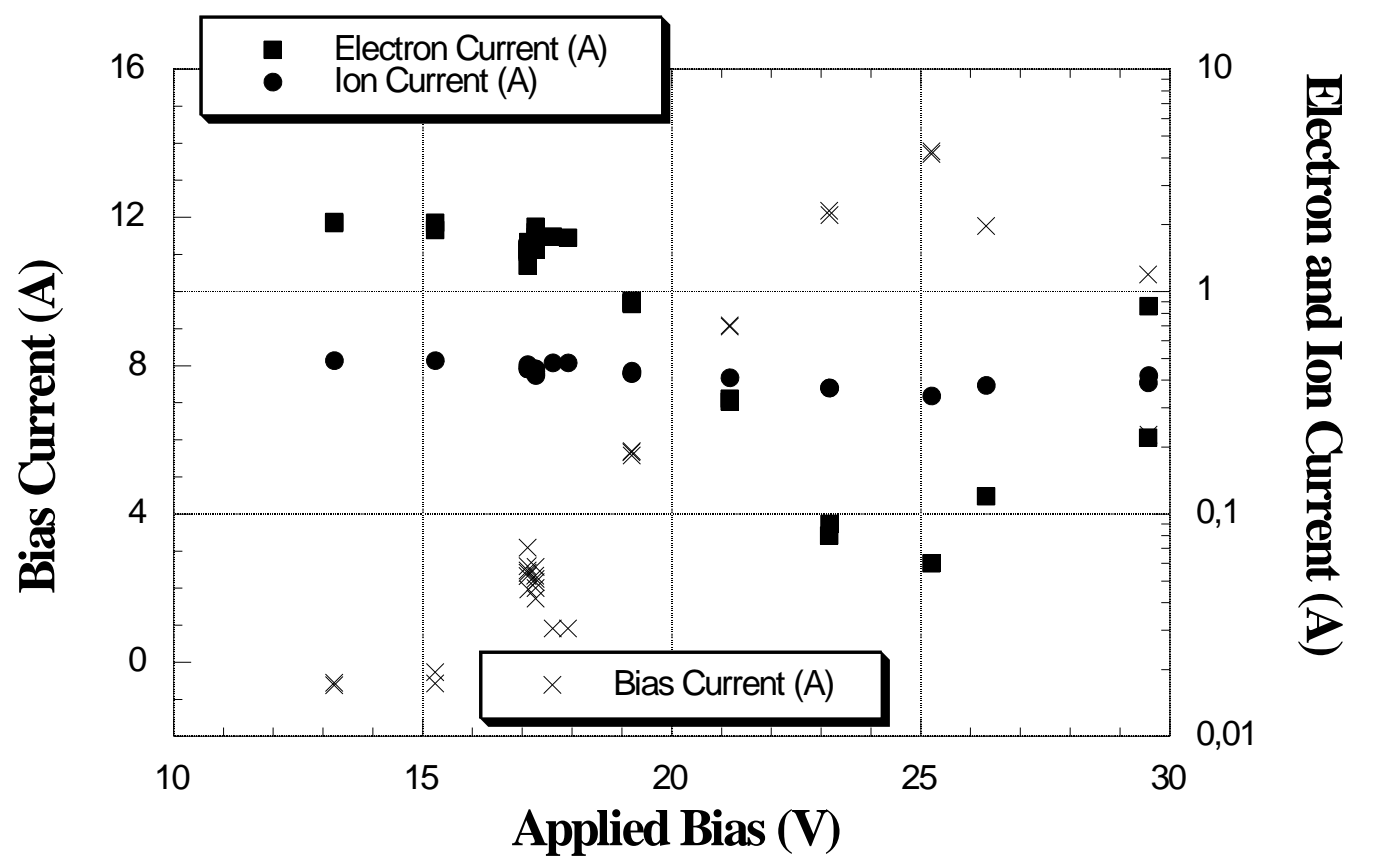

FIG. 9 